Pacific Journal of Mathematic 


\section{A STUDY OF MULTIVALUED FUNCTIONS}

Carlos J. R. Borges

The primary purpose of this study is to determine which topological properties of a space are preserved by multivalued functions. Among other results, the following are proved:

(A) Let $F: X \rightarrow Y$ be a perfect map from $X$ onto $Y$, with $F(x) \neq \varnothing$ for each $x \in X$, where $X$ and $Y$ are $T_{1}$-spaces whose diagonals are $G_{\delta}$-sets. Then $X$ is metrizable (stratifiable) if and only if $Y$ is metrizable (stratifiable)-see Theorem 3.2.

(B) If $F: X \rightarrow Y$ is a multivalued $Y$-compact quotient map from a separable metrizable space $X$ onto a regular first countable space $Y$ with a $G_{\delta}$-diagonal, then $Y$ is separable metrizable (see Theorem 4.5).

(C) Every (usc-) lsc-function $F$ from a closed subset of a stratifiable space $X$ to a topological space $Y$ admits a (usc-) lsc-extension to all of $X$ (see Theorem 5.2).

Multivalued functions have been extensively studied by Kruse [6], Michael $[7 ; 8]$, Ponomarev $[12 ; 13 ; 14]$, Smithson [15] and Strother $[17 ; 18]$. Choquet [2] and Hahn [3] have also considered multivalued functions.

2. Preliminary definitions and results. Because there are many conflicting terminologies in the theory of multivalued functions, we find it necessary to attempt a terminology of our own, which is a direct extension of the most natural and simple terminology of Michael [10] and includes some of Ponomarev's terminology:

Definition 2.1. For any sets $X$ and $Y, F: X \rightarrow Y$ is a multivalued function provided that, for each $x \in X, F(x)$ is a subset of $Y(F(x)$ need not be a closed or nonempty set as required by Ponomarev and others).

Clearly, single-valued functions are just special cases of multivalued functions and indeed a multivalued function from $X$ to $Y$ can obviously be thought of as a single-valued function from $X$ to $\mathscr{A}(Y)$-the family of all subsets of $Y$ (including the empty set).

Definition 2.2. Let $F: X \rightarrow Y$ be a multivalued function. Then

(a) $F(A)=\bigcup\{F(x) \mid x \in A\}$ for each $A \subset X$,

(b) $F^{-1}(B)=\{x \in X \mid F(x) \cap B \neq \varnothing\}$ for each $B \subset Y$ (clearly $F^{-1}$ is a multivalued function from $Y$ to $X$ ).

It is quite easy to construct a multivalued function $F$ from a 
topological space $X$ to a topological space $Y$ such that $F^{-1}(U)$ is open for each open $U \subset Y$ and yet $F^{-1}(B)$ is not closed for each closed $B \subset Y$ and vice versa. Hence on immediately realizes that the various equivalent definitions of continuity of (single-valued) functions have to be considered separately for multivalued functions:

Definition 2.3. Let $F: X \rightarrow Y$ be a multivalued function. Then we say that (the terminology of $(\mathrm{e})-(\mathrm{g})$ was first developed by Ponomarev [13])

(a) $F$ is a usc-function (i.e., upper semi-continuous function) provided that $F^{-1}(B)$ is closed for each closed $B \subset Y$,

(b) $F$ is a lsc-function (i.e., lower semi-continuous function) provided that $F^{-1}(V)$ is open for each open $V \subset Y$,

(c) $F$ is a continuous function provided that $F$ is a usc-function and a lsc-function,

(d) $F$ is a closed (open) function provided that $F(B)$ is closed (open) for each closed (open) $B \subset X$,

(e) $F$ is $Y$-compact ( $Y$-separable) ( $X$-compact: $X$-separable) provided that $F(x)$ is compact (separable) for each $x \in X\left(F^{-1}(y)\right.$ is compact for each $y \in Y ; F^{-1}(y)$ is separable for each $\left.y \in Y\right)$,

(f) $F$ is $Y$-perfect ( $X$-perfect) provided that $F$ is a closed, $Y$ compact, usc-function ( $F$ is a closed, $X$-compact, usc-function),

(g) $F$ is perfect provided that $F$ is $X$-perfect and $Y$-perfect.

Our terminology compares with others as follows (where "三" means "the same as"):

1. usc $\equiv$ strong upper semi-continuity (Choquet [2]) $\equiv$ upper semicontinuous (Michael [7]) $\equiv$ continuous (Ponomarev [12]) $\equiv$ lower continuous (Hahn [3]).

2. lsc $\equiv$ strong lower semi-continuity (Choquet [2]) $\equiv$ lower semicontinuous (Michael [7]) $\equiv$ skew-continuous (Ponomarev [12]) $\equiv$ lower continuous (Hahn [3]).

Definition 2.4. Let $F: X \rightarrow Y$ be a multivalued function. Then (a) The graph of $F$ is

$$
\operatorname{gr} F=\{(x, y) \in X \times Y \mid y \in F(x) \text { and } x \in X\} .
$$

(b) The functions $p_{X}: g r F \rightarrow X$ and $p_{Y}: g r F \rightarrow Y$ are defined by

$$
p_{X}(x, y)=x \text { and } p_{Y}(x, y)=y
$$

for each $(x, y) \in g r F$.

We now state, without proof, some straightforward results of crucial importance which were first observed by Ponomarev and Smirnov. 
Lemma 2.5. Let $F: X \rightarrow Y$ be a multivalued function from $X$ onto $Y$. Then

(a) $\left(F^{-1}\right)^{-1}=F$,

(b) $F(x)=p_{Y} p_{X}^{-1}(x)$ for each $x \in X$,

(c) $F^{-1}(y)=p_{X} p_{Y}^{-1}(y)$ for each $y \in Y$,

(d) the maps $p_{X}$ and $p_{Y}$ are always continuous (since they are restrictions of the projection maps $\pi_{X}: X \times Y \rightarrow X$ and $\pi_{Y}: X \times Y \rightarrow Y$ ).

We now prove two very useful results which determine the relationship of a multivalued map $F: X \rightarrow Y$ and the maps $p_{X}$ and $p_{Y}$. The proof of the first result is essentially due to Smirnov.

THEOREM 2.6. Let $X$ and $Y$ be topological spaces and $F: X \rightarrow Y$ be a multivalued function. Then

(a) $F$ is a usc-function and Y-compact if and only if the map $p_{X}$ is perfect,

(b) $F$ is closed and $X$-compact if and only if the map $p_{Y}$ is perfect,

(c) $F$ is perfect if and only if $p_{X}$ and $p_{Y}$ are perfect.

Proof. The proof of the "only if" part of (a) appears in the footnote of page 123 of Ponomarev [13]. The proof of the "if" part of (a) follows easily from Lemma 2.5 and Definition 2.4(a). The proof of (b) is the same as the proof of (a) if one considers $F^{-1}$ instead of $F$. Part (c) is an immediate consequence of parts (a) and (b).

REMARK 2.7. The appealing conjecture that $p_{X}\left(p_{Y}\right)$ is closed even though $F$ is not $Y$-compact ( $X$-compact) is false: Let $R$ be the real line with the usual topology, $I$ be the closed unit interval and $F: R \rightarrow R$ be defined by $F(x)=I$ for each $x \in R$. Then $F$ is not $X$-compact $\left(F^{-1}(0)=R\right)$ and $p_{Y}$ is not a closed map $\left(p_{Y}\left(\left\{(x, y) \mid y=e^{x}\right.\right.\right.$ and $x \leqq 0\})=[0,1])$. Furthermore, $F$ is $Y$-compact, continuous and closed.

THEOREM 2.8. Let $F: X \rightarrow Y$ be a multivalued function. Then

(a) if $F$ is a lsc-function then $p_{X}$ is an open map,

(b) if $F$ is open then $p_{Y}$ is open.

Proof. It suffices to prove (a), since (b) is an immediate consequence of (a), due to Definition 2.4(b) and Lemma 2.5(a): Therefore, let us assume $p_{X}$ is not open. Then there exists a closed $A \subset g r F$ such that the set $B=\left\{x \in X \mid p_{X}^{-1}(x) \subset A\right\}=X-p_{X}(g r F-A)$ is not a closed subset of $X$. Let $w \in B^{-}-B$. Then $p_{X}^{-1}(w) \not \subset A$. Let $(w, z) \in p_{X}^{-1}(w)-A$. Since $A$ is a closed subset of $g r F$ there exists an 
open neighborhood $N=U \times X$ of $(w, z)$ in $X \times Y$ such that $N \cap A=$ $\varnothing$. Letting

$$
C=X-F^{-1}(V)=\{x \in X \mid F(x) \cap V=\varnothing\}
$$

we get that $C$ is not a closed subset of $X$, since $w \in U-C(z \in F(w) \cap V)$, $w \in U \cap B^{-}$and $\varnothing \neq U \cap B \subset C$ (Suppose there exists $x \in(U \cap B)-C$. Then $V \subset F(x) \neq \varnothing$ and thus

$$
\varnothing \neq(U \times V) \cap(\{x\} \times F(x))=(U \times V) \cap p_{X}^{-1}(x) \subset N \cap A=\varnothing,
$$

a contradiction). Hence $F^{-1}$ is clearly not an open function (i.e., $F$ is not a lsc-function), a contradiction which completes the proof.

Finally we prove a rather interesting result (even though we cannot find it in the literature, it appears to be known).

THEOREM 2.9. The relation between spaces $X$ and $Y$ expressed by "there is a perfect multivalued function $F$ from $X$ onto $Y$ such that $F(x) \neq \varnothing$ for each $x \in X$ " is an equivalence relation.

Proof. The only difficult part of the proof involves showing that the composition of a $Y$-compact usc-function $F: X \rightarrow Y$ and a $Z$-compact usc-function $G: Y \rightarrow Z$ is $Z$-compact; but this is an immediate consequence of Corollary 9.6 of Michael [10] (see also 7.7 (p. 60) of Kruse [6], Th. 1 (p. 104) of Ponomarev [12] and 21.3.4 of Hahn [3]).

3. Preservation of topological properties. First we will prove necessary and sufficient conditions for the preservation of metrizability and stratifiability ${ }^{1}$ by multivalued functions, for which we need the following lemma:

Lemma 3.1. Let $X$ and $Y$ be topological spaces with $G_{\delta}$-diagonals. ${ }^{2}$ Then $X \times X$ has a $G_{\delta}$-diagonal.

Proof. Suppose that $U_{n}$ and $V_{n}$ are open in $X \times X$ and $Y \times Y$ respectively, and $\bigcap_{n=1}^{\infty} U_{n}$ and $\bigcup_{n=1}^{\infty} V_{n}$ are the diagonals of $X \times X$ and $Y \times Y$ respectively. Let

$$
W_{n}=\left\{\left(\left(x_{1}, y_{1}\right),\left(x_{2}, y_{2}\right)\right) \mid\left(x_{1}, x_{2}\right) \in U_{n} \text { and }\left(x_{2}, y_{2}\right) \in V_{n}\right\} \text {. }
$$

Then $W_{n}$ is open in $(X \times Y) \times(X \times Y)$ and $\bigcup_{n=1}^{\infty} W_{n}$ is the diagonal of $(X \times Y) \times(X \times Y)$.

1 A summary of the properties of stratifiable spaces appears in the introduction of [1]. The most relevant results about these spaces which should be mentioned here are (a) Every $C W$-complex of Whitehead is stratifiable, (b) metrizability implies stratifiability which implies paracompactness and perfect normality.

2 A topological space $X$ has a $G_{\delta}$-diagonal if $\{(x, x) \mid x \in X\}$ is a $G_{\delta^{-}}$subset of $X \times X$. 
THEOREM 3.2. Let $F: X \rightarrow Y$ be a perfect multivalued function, with $F(x) \neq \varnothing$ for each $x \in X$ (i.e., $\left.F^{-1}(Y)=X\right)$, where $X$ and $Y$ are $T_{1}$-spaces with G-diagonals. Then $X$ is metrizable (stratifiable) if and only if $Y$ is metrizable (stratifiable).

Proof. We will first prove the "only if" part: Let $X$ be metrizable (stratifiable) and $Y$ have a $G_{\delta}$-diagonal. Then $g r F$ has a $G_{\delta}$-diagonal (a subspace of a space with a $G_{\delta}$-diagonal clearly has a $G_{\delta}$-diagonal) and hence $g r F$ is metrizable (stratifiable) because of Theorem 8.1 (Theorem 8.4) of [1].

Since $p_{Y}$ is perfect, due to Theorem 2.6(c), we get that $Y$ is metrizable (stratifiable) due to Theorem 1 of Stone [16] (Th. 3.1 of [1]). The "if" part follows immediately from Theorem 2.9.

We will now turn our attention to the preservation of various other topological properties by multivalued maps, for which we will need the following definition:

Definition 3.3. Let $F: X \rightarrow Y$ be a multivalued map. Then $F$ is said to be $Y$-monotone (monotone) provided that each $F(x)$ is connected (each $F^{-1}(y)$ is connected).

THEOREM 3.4. Let $F: X \rightarrow Y$ be an onto (i.e., $F(x)=Y$ ) multivalued function such that $F(x) \neq \varnothing$ for each $x \in X$. Then

(a) if $F$ is a lsc-function, $F(x)$ is separable for each $x \in X$ and $X$ is separable then $Y$ is separable,

(b) if $F$ is a $Y$-monotone lsc-function and $X$ is connected then $Y$ is connected.

Proof. (a) By Lemma 2.8(a), $p_{X}$ is an open function and hence $g r F$ is easily seen to be separable. Hence $Y$ is separable ( $p_{Y}$ is continuous).

(b) Clearly, it suffices to prove that $g r F$ is connected: Assume not. Then $g r F$ is the union of two disjoint nonempty open subsets $U$ and $V$. Since $P_{X}$ is clearly monotone we get that $p_{X}^{-1}(x) \cap U \neq \varnothing$ if and only if $p_{X}^{-1}(x) \subset U$. Hence $\left\{p_{X}(U), p_{X}(V)\right\}$ is an open disjoint cover of $X$, because of Lemma 2.8(a), a contradiction.

As pointed out by the referee, both parts of the preceding result become false if the hypothesis that $F(x) \neq \varnothing$, for each $x \in X$, is removed (Example (a). Let $Z$ be a countably infinite discrete space, $Y=(\beta Z)-Z(\beta Z$ denotes the $\beta$-compactification of $Z)$ and define $F: \beta F \rightarrow Y$ by $F(x)=\{x\} \cap Y$. Clearly $F$ is a perfect $Y$-separable map. However, while $\beta Z$ is clearly separable it is well-known that $Y$ is not separable (see, for example, exercise $60(1,2)$ of [4] for an 
easy proof of the fact that $Y$ has $2^{\aleph_{0}}$ mutually disjoint open subsets which clearly implies that $Y$ is not separable). Example (b). Let $X=[0,2], Y=X-\{1\}$ and define $F: X \rightarrow Y$ by $F(x)=\{x\} \cap Y)$.

As a final comment of this section, we note that the requirement that $X$ and $Y$ be completely regular in the statement of Theorem 3 (page 127) of Ponomarev [13] is much stronger than needed, as the ensuing result indicates:

Theorem 3.5. Let $X$ and $Y$ be regular spaces and $F: X \rightarrow Y$ be an onto perfect multivalued function. If $X$ is paracompact (locally compact; countably paracompact; star-paracompact) then so is $Y$. The converse is also true if $F(x) \neq \varnothing$ for each $x \in X$.

Proof. Since $F$ is a usc-function then $F^{-1}(X)$ is a closed subset of $X$. Hence the "if" part follows immediately from Theorem 2.2 of [5] and Theorem 1 of [11], whose proofs clearly depend only on the fact that $X$ and $Y$ are regular spaces, and Theorem 2.6. The converse follows from Theorem 2.10.

4. Multivalued quotient maps. Since multivalued functions behave very much like single-valued functions, it seems imperative that one consider the extension of a quotient map for single-valued functions to multivalued functions. In so doing, one is immediately compelled to consider two distinct concepts of multivalued quotient maps in the same manner as with the continuity of multivalued maps. However, it turns out that the situation is not as simple as it seems. Therefore, let us first prove some results which will justify our Definition 4.2 .

Lemma 4.1. Let $F: X \rightarrow Y$ be a multivalued function and let $X$ be a topological space. Then

(a) $\left\{U \subset Y \mid F^{-1}(U)\right.$ is open $\}$ is not necessarily a base for a topology on $Y$.

(b) $\left\{U \subset Y \mid F^{-1}(Y-U)\right.$ is closed $\}$ is a base for a topology on $Y$.

Proof. It is easily seen that

(i) $F^{-1}\left(\bigcup_{\alpha \in \Lambda} A_{\alpha}\right)=\bigcup_{\alpha \in A} F^{-1}\left(A_{\alpha}\right)$

(ii) $F^{-1}\left(\bigcup_{\alpha \in \Lambda} A_{\alpha}\right) \subset \bigcup_{\alpha \in \Lambda} F^{-1}\left(A_{\alpha}\right)$, and equality does not necessarily hold, for any collection $\left\{A_{\alpha}\right\}_{\alpha \in \Lambda}$ of subsets of $Y$.

In order to prove (a) let $X$ be the real line with the usual topology, $Y$ the set of all real numbers, and let $F: X \rightarrow Y$ be defined by

$$
\begin{aligned}
& F(1)=[1,2], \\
& F(x)=Y-[1,2] \text { for all } x \neq 1 .
\end{aligned}
$$


Then $F^{-1}(\{y \in Y \mid y \geqq(3 / 2)\})$ and $F^{-1}(\{y \in Y \mid y \leqq(3 / 2)\})$ are open subsets of $X$, but $F^{-1}(\{3 / 2\})$ is not an open subset of $X$. Hence $\left\{U \subset Y \mid F^{-1}(U)\right.$ is open $\}$ is not a base for a topology on $Y$.

Finally we prove (b): Let $y \in U \cap V$ such that $F^{-1}(Y-U)$ and $F^{-1}(Y-V)$ are closed subsets of $X$. Since

$$
\begin{aligned}
F^{-1}(Y-V \cap U) & =F^{-1}((Y-U) \cup(Y-V)) \\
& =F^{-1}(Y-U) \cup F^{-1}(Y-V),
\end{aligned}
$$

due to (i), one easily sees that $\left\{U \subset Y \mid F^{-1}(Y-U)\right.$ is closed $\}$ is a base for a topology on $Y$, which completes the proof.

While Lemma 4.1 dashes all hopes of developing a concept of "multivalued quotient topology," nonetheless one can define a multivalued quotient map $F: X \rightarrow Y$ in such a way that $F$ is either a usc-function or a lsc-function.

Definition 4.2. Let $X$ and $Y$ be topological spaces and $F: X \rightarrow Y$ an onto multivalued map. Then $F$ is said to be a us-quotient (lsquotient) map provided that a subset $U$ of $Y$ is closed (open) if and only if $F^{-1}(U)$ is a closed subset of $X\left(F^{-1}(U)\right.$ is an open subset of $\left.X\right)$. $F$ is said to be a quotient map whenever $F$ is both a us-quotient map and a $l s$-quotient map.

We will now study the relationship between a multivalued function $F: X \rightarrow Y$ and the maps $p_{X}$ and $p_{Y}$, as well as other relations.

Proposition 4.3. Let $X$ and $Y$ be topological spaces and $F: X \rightarrow Y$ a multivalued function. Then

(a) if $p_{X}$ is closed and $F$ is a $u s$-quotient map then $p_{Y}$ is a quotient map,

(b) if $F$ is a $l s$-quotient map then $p_{Y}$ is a quotient map, and $p_{X}$ is an open map,

(c) if $F$ is a $Y$-compact us-quotient map then $p_{Y}$ is a quotient map, and $p_{X}$ is a perfect map.

Proof. Part (a) is straightforward by use of Lemma 2.5(c). Part (b) is straightforward by use of Lemma 2.5(c) and Theorem 2.8(a). Part (c) is an immediate consequence of (a) and Theorem 2.6(a).

Now we generalize Theorem 3 and the Corollary on page 695 of Stone [16] to multivalued quotient maps.

THEOREM 4.4. Let $F: X \rightarrow Y$ be a multivalued $Y$-compact quotient map from the locally compact separable metrizable space $X$ onto a Hausdorff first countable space $Y$ with a $G_{\delta}$-diagonal. Then $Y$ is a 
locally compact separable metrizable space.

Proof. By Proposition 4.3, $p_{Y}$ is a quotient map and $p_{X}$ is an open perfect map. Hence $g r F$ is locally compact (due to Theorem 3.5), metrizable (due to Theorem 3.2), and separable (because $p_{X}$ is open and each $p_{X}^{-1}(x)$ is separable-indeed each $p_{X}^{-1}(x)$ is compact metrizable). Since $p_{Y}$ is a quotient map, we get that $Y$ is a locally compact separable meirizable space, by Theorem 3 of [16].

Similarly one can prove the following result, by using the Corollary of page 695 of [16]:

THEOREM 4.5. Let $F: X \rightarrow Y$ be a multivalued $Y$-compact quotient map from the separable metrizable space $X$ onto a regular first countable $T_{1}$-space $Y$ with a $G_{\delta}$-diagonal. Then $Y$ is a separable metrizable space.

Finally we generalize Theorem 4 of Stone [16] to multivalued open maps.

THEOREM 4.6. Let $F: X \rightarrow Y$ be an open, $Y$-compact, $X$-separable continuous function from a metrizable and locally separable space $X$ onto a regular $T_{1}$-space $Y$ with a $G_{\delta}$-diagonal. Then $Y$ is metrizable and locally separable.

Proof. Similar to the proof of Theorem 4.4 except that we use, respectively, Theorem 2.8(b), Theorem 2.8(a) and 2.6(a), and Theorem 4 of [16].

5. Continuous extensions. We will now generalize Theorem 4.3 of [1] to multivalued functions. Throughout this section, we let $C_{u}(Y, Z)\left(C_{l}(Y, Z) ; C(Y, Z)\right)$ be the space of all usc-functions (lscfunctions; continuous functions) from $Y$ to $Z$, for any topological spaces $Y$ and $Z$.

Before stating our main theorem we state the following straightforward but crucial result:

Lemma 5.1. Let $F: X \rightarrow Y$ be a multivalued function. Then

(a) $F$ is a usc-function if and only if for each $x \in X$ and neighborhood $V$ of $F(x)$ there exists a neighborhood $U$ of $x$ such that $F(U) \subset V$.

(b) $F$ is a lsc-function if and only if for each $x \in X$ and open $V \subset Y$ such that $F(x) \cap V \neq \varnothing$ there exists a neighborhood $U$ of $x$ such that $F(z) \cap V \neq \varnothing$ for each $z \in U$. 
THeOREM 5.2. Let $X$ be a stratifiable space, $A$ a closed subset of $X$ and $E$ any topological space. Then there exist mappings

$$
\begin{aligned}
& \varphi: C_{u}(A, E) \rightarrow C_{u}(X, E), \\
& \psi: C_{l}(A, E) \rightarrow C_{l}(X, E),
\end{aligned}
$$

satisfying the following conditions:

(a) each $\varphi(f)$ and $\varphi(f)$ is an extension of $f$,

(b) range $\varphi(f)=$ range $f$; range $\psi(f)=$ range $f$,

(c) $\varphi(f)(x) \neq \varnothing(\psi(g)(x) \neq \varnothing)$ for each $x \in X$ whenever $f(x) \neq \varnothing$ $(g(x) \neq \varnothing)$ for each $x \in A$.

Proof. Without further comment we will frequently use the terminology of Definition 4.1 of [1] and the crucial results of Lemma 4.2 of [1], since our proof parallels that of Theorem 4.3 of [1], to a large extent. Let $W=X-A$, and let $W^{\prime}=\left\{x \in W \mid x \in U_{y}\right.$ for some $y \in A$ and open $U$ containing $y\}$. For each $x \in W^{\prime}$, let

$$
m(x)=\max \left\{n(U, y) \mid y \in A \text { and } x \in U_{y}\right\} .
$$

It is easily seen that each $m(x) \leqq n(W, x)<\infty$.

Using the paracompactness of $W$, let $\mathscr{V}$ be an open locally finite (with respect to $W$ ) cover of $W$ such that $\left\{V^{-} \mid V \in \mathscr{Y}^{-}\right.$and $V^{-}$is the closure of $V$ with respect to $W\}$ is a refinement of $\left\{W_{x} \mid x \in W\right\}$. For each $V \in \mathscr{Y}^{-}$, pick $x_{V} \in W$ such that $V^{-} \subset W_{x_{V}}$. If $x_{V} \in W^{\prime}$, pick $a_{V} \in A$ and open $S_{V}$ containing $a_{V}$ such that $x_{V} \in\left(S_{V}\right)_{a_{V}}$ and $n\left(S_{V}, a_{V}\right)=$ $m\left(x_{V}\right)$; if $x_{V} \notin W^{\prime}$, let $a_{V}$ be the fixed point $a_{0} \in A$.

We now define two functions $g: X \rightarrow E$ and $h: X \rightarrow E$ by

$$
\begin{array}{ll}
g(x)=f(x)=h(x) & \text { if } x \in A, \\
g(x)=\bigcup\left\{f\left(a_{V}\right) \mid x \in V^{-}\right\} & \text {if } x \in W, \\
h(x)=\bigcup\left\{f\left(a_{V}\right) \mid x \in V\right) & \text { if } x \in W .
\end{array}
$$

It is easily seen that $g$ is a usc-function on $W$ (Let $x \in W$ and let $V$ be a neighborhood of $g(x)$. Then $U=W-\mathbf{U}\left\{V^{-} \mid V \in \mathscr{V}^{\text {- }}\right.$ and $\left.x \notin V-\right\}$ is a neighborhood of $x$, and clearly $g(U) \subset V$; indeed, for each $z \in U$, $g(z) \subset g(x))$. Similarly, one easily sees that $h$ is a lsc-function on $W$ (Let $x \in W$ and let $U$ be an open subset of $E$ such that $h(x) \cap U \neq \varnothing$. By the definition of $h(x)$ there exists some $V \in \mathscr{V}^{-}$such that $f\left(a_{V}\right) \cap U \neq \varnothing$ and $x \in V$. Then $h(z) \cap U \neq \varnothing$ for each $\left.z \in V\right)$.

To show that $g$ is a usc-function at $b \in A$, whenever $f \in C_{u}(A, E)$, let 0 be any open subset of $E$ containing $f(b)$. Since $f$ is a uscfunction, there exists an open neighborhood $N$ of $b$ such that $f(A \cap N) \subset 0$.

Now it is not hard to see that 
(a) If $x \in\left[\left(N_{b}\right)_{b}-A\right] \cap V^{-}$with $V \in \mathscr{V}^{-}$and $V^{-}$the closure of $V$ with respect to $W$, then $a_{V} \in N$. (A detailed proof of (a) can be found in the last paragraph of the proof of Theorem 4.3 of [1].) Consequently, $g\left(\left(N_{b}\right)_{b}\right) \subset 0$, due to $(a)$, and thus $g$ is a usc-function.

To show that $h$ is a lsc-function at $b \in A$, whenever $f \in C_{l}(A, E)$, let 0 be any open subset of $E$ such that $f(b) \cap 0 \neq \varnothing$. Since $f$ is a lsc-function, there exists an open neighborhood $N$ of $b$ such that $f(z) \cap 0 \neq \varnothing$ for each $z \in N \cap A$. It is easily seen that $\left.x \in\left(N_{b}\right)_{b}\right)-A$ implies $h(x) \cap 0 \neq \varnothing$, due to (a) and the definition of $h(x)$. Consequently, $h(x) \cap 0 \neq \varnothing$ for each $x \in\left(N_{b}\right)_{b}$, and thus $h$ is a lsc-function.

To complete the proof, we simply let $\varphi(f)=g$ and $\psi(f)=h$.

REMARK 5.3. It is easily seen that the function $g$ is not necessarily a lsc-function on $W$ (Let $U$ be any subset of $E$ and suppose $f\left(a_{V}\right) \cap U \neq \varnothing$ for some $V \in \mathscr{Y}^{-}$. Then $g(x) \cap U \neq \varnothing$ for each $x \in V^{-}$, since $x \in V^{-}$implies $f\left(a_{V}\right) \subset g(x)$ by the definition of $g(x)$. Hence

$$
g^{-1}(U)=\bigcup\left\{V^{-1} \mid f\left(a_{V}\right) \cap U \neq \varnothing\right\}
$$

is a closed subset of $W$ for any subset $U$ of $E$ (since $\mathscr{V}^{-}$is locally finite with respect to $W)$ ). Thus $g$ is not a lsc-function, unless $g^{-1}(U)$ is clopen ${ }^{3}$ for each open subset $U$ of $E$. We have thus essentially proved the following result:

THeorem 5.4. Let $X$ be a stratifiable space, $A$ a closed subset of $X$ such that $\operatorname{dim}(X-A)=0$ (covering dimension). Then there exist mappings

$$
\begin{aligned}
& \varphi: C_{u}(A, E) \longrightarrow C_{u}(X, E) \\
& \psi: C_{l}(A, E) \longrightarrow C_{l}(X, E) \\
& \theta: C(A, E) \longrightarrow C(X, E)
\end{aligned}
$$

satisfying the following

(a) Each $\varphi(f), \psi(f)$ and $\theta(f)$ is an extension of $f$,

(b) range $\varphi(f)=$ range $\psi(f)=$ range $\theta(f)=$ range $f$.

Proof. The same as the proof of Theorem 5.2 except that we choose the open cover $\mathscr{V}^{-}$of $X-A$ so that each $V \in \mathscr{Y}^{-}$is a clopen subset of $X-A$ (see Proposition 2(b) of [9]).

We end this section with the following two questions:

1. Does Theorem 5.2 remain valid if $X$ is a paracompact space? (We conjecture a negative answer.)

${ }^{3}$ A subset $U$ of a topological space $X$ is said to be clopen provided that $U$ is an open and closed subset of $X$. 
2. In Theorem 5.4, does the existence of the mapping $\theta$ imply that for some single-valued quotient map $q$ from $X$ onto $\tilde{X}$, say, $q$ maps $A$ homomorphically onto a closed subset of $\tilde{X}, q(A)$ and $q(X-A)$ are disjoint, and $\operatorname{dim}(q(X-A))=0$ ?

\section{BIBLIOGRAPHY}

1. C. J. R. Borges, On stratifiable spaces, Pacific J. Math. 17 (1966), 1-16.

2. G. Choquet, Convergences, Annales de l'Université de Grenoble 23 (1947-1948), 55112.

3. H. Hahn, Reele Funktionen, Akademische Verlagsgesellschaft, Leipzig, 1932.

4. Gillman and Gerison, Rings of Continuous Functions, Van Nostrand, 1960.

5. M. Henriksen and J. Isbell, Some properties of compactifications, Duke Math. J. 25 (1958), 83-105.

6. A. H. Kruse, Introduction to the theory of block assemblages and related topics in topology, University of Kansas, 1956 (NSF-sponsored Technical Report).

7. E. A. Michael, Continuous Selections I, Ann. of Math. 63 (1956), 361-382.

8. Continuous Selections II, Ann. of Math. 64 (1956), 562-580.

9. - Selected selection theorems, Amer. Math. Monthly 68 (1956), 233-238.

10. Topologies on spaces of subsets, Trans. Amer. Math. Soc. 71 (1951), 152182.

11. K. Nagami, A note on Hausdorff spaces with the star-finite property, II, Proc. Japan Acad. 37 (1961), 189-192.

12. V. I. Ponomarev, A new space of closed sets and multivalued continuous mappings of bicompacta, Amer. Math. Soc. Transl. (2) 38 (1964), 95-118.

13. - Properties of topological spaces preserved under multivalued continuous mappings, Amer. Math. Soc. Transl. (2) 38 (1964), 119-140.

14. - On the extension of multivalued mappings of topological spaces to their compactifications, Amer. Math. Soc. Transl. (2) 38 (1964), 141-158.

15. R. E. Smithson, Some general properties of multivalued functions, Pacific J. Math. 15 (1965), 681-703.

16. A. H. Stone, Metrizability of decomposition spaces, Proc. Amer. Math. Soc. 7 (1956), 690-700.

17. W. Strother, Continuous multivalued functions, Boletin da Sociedade de S. Paulo 10 (1958), 87-120.

18. - On an open question concerning fixed points, Proc. Amer. Math. Soc. 4 (1953), 988-993.

Received September 21, 1966, and in revised form March 30, 1967. This research was supported by a University of California Faculty Fellowship.

University of CALIFornia, DaVis 



\section{PACIFIC JOURNAL OF MATHEMATICS}

\section{H. ROYDEN}

Stanford University

Stanford, California

\author{
J. P. JANS \\ University of Washington \\ Seattle, Washington 98105
}

\section{EDITORS}

\author{
J. DugundJI \\ Department of Mathematics \\ Rice University \\ Houston, Texas 77001 \\ Richard ARENS \\ University of California \\ Los Angeles, California 90024
}

\section{ASSOCIATE EDITORS}
E. F. BECKENBACH
B. H. NeumanN
F. WOLF
K. YOSIDA

\section{SUPPORTING INSTITUTIONS}

\author{
UNIVERSITY OF BRITISH COLUMBIA \\ CALIFORNIA INSTITUTE OF TECHNOLOGY \\ UNIVERSITY OF CALIFORNIA \\ MONTANA STATE UNIVERSITY \\ UNIVERSITY OF NEVADA \\ NEW MEXICO STATE UNIVERSITY \\ OREGON STATE UNIVERSITY \\ UNIVERSITY OF OREGON \\ OSAKA UNIVERSITY \\ UNIVERSITY OF SOUTHERN CALIFORNIA
}

\author{
STANFORD UNIVERSITY \\ UNIVERSITY OF TOKYO \\ UNIVERSITY OF UTAH \\ WASHINGTON STATE UNIVERSITY \\ UNIVERSITY OF WASHINGTON \\ AMERICAN MATHEMATICAL SOCIETY \\ CHEVRON RESEARCH CORPORATION \\ TRW SYSTEMS \\ NAVAL ORDNANCE TEST STATION
}

Mathematical papers intended for publication in the Pacific Journal of Mathematics should be typewritten (double spaced). The first paragraph or two must be capable of being used separately as a synopsis of the entire paper. It should not contain references to the bibliography. Manuscripts may be sent to any one of the four editors. All other communications to the editors should be addressed to the managing editor, Richard Arens at the University of California, Los Angeles, California 90024.

50 reprints per author of each article are furnished free of charge; additional copies may be obtained at cost in multiples of 50 .

The Pacific Journal of Mathematics is published monthly. Effective with Volume 16 the price per volume (3 numbers) is $\$ 8.00$; single issues, $\$ 3.00$. Special price for current issues to individual faculty members of supporting institutions and to individual members of the American Mathematical Society: $\$ 4.00$ per volume; single issues $\$ 1.50$. Back numbers are available.

Subscriptions, orders for back numbers, and changes of address should be sent to Pacific Journal of Mathematics, 103 Highland Boulevard, Berkeley 8, California.

Printed at Kokusai Bunken Insatsusha (International Academic Printing Co., Ltd.), 7-17, Fujimi 2-chome, Chiyoda-ku, Tokyo, Japan.

PUBLISHED BY PACIFIC JOURNAL OF MATHEMATICS, A NON-PROFIT CORPORATION

The Supporting Institutions listed above contribute to the cost of publication of this Journal, but they are not owners or publishers and have no responsibility for its content or policies. 


\section{Pacific Journal of Mathematics}

A. A. Aucoin, Diophantine systems ............................. 419

Charles Ballantine, Products of positive definite matrices. I ............... 427

David Wilmot Barnette, A necessary condition for d-polyhedrality ............ 435

James Clark Beidleman and Tae Kun Seo, Generalized Frattini subgroups of finite groups ......................................... 441

Carlos Jorge Do Rego Borges, A study of multivalued functions ............. 451

William Edwin Clark, Algebras of global dimension one with a finite ideal

lattice ...............................................

Richard Brian Darst, On a theorem of Nikodym with applications to weak convergence and von Neumann algebras .........................

George Wesley Day, Superatomic Boolean algebras .....................

Lawrence Fearnley, Characterization of the continuous images of all

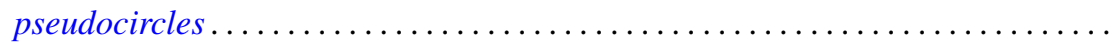

Neil Robert Gray, Unstable points in the hyperspace of connected subsets....... 515

Franklin Haimo, Polynomials in central endomorphisms .................. 521

John Sollion Hsia, Integral equivalence of vectors over local modular lattices . . . . 527

Jim Humphreys, Existence of Levi factors in certain algebraic groups .......... 543

E. Christopher Lance, Automorphisms of postliminal $C^{*}$-algebras ............ 547

Sibe Mardesic, Images of ordered compacta are locally peripherally metric . . . . 557

Albert W. Marshall, David William Walkup and Roger Jean-Baptiste Robert Wets,

Order-preserving functions: Applications to majorization and order

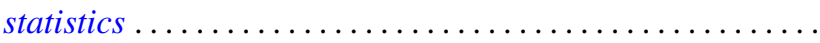

Wellington Ham Ow, An extremal length criterion for the parabolicity of

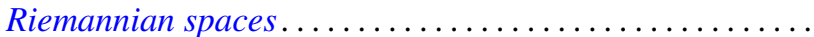

585

Wellington Ham Ow, Criteria for zero capacity of ideal boundary components of

Riemannian spaces...................................... 591

J. H. Reed, Inverse limits of indecomposable continua .................. 597

Joseph Gail Stampfli, Minimal range theorems for operators with thin spectra . . . 601

Roy Westwick, Transformations on tensor spaces..................... 613

Howard Henry Wicke, The regular open continuous images of complete metric

spaces ........................................... 621

Abraham Zaks, A note on semi-primary hereditary rings .............. 627

Thomas William Hungerford, Correction to: "A description of $\operatorname{Mult}_{i}\left(A^{1}, \cdots, A^{n}\right)$

by generators and relations" ............................. 629

Uppuluri V. Ramamohana Rao, Correction to: "On a stronger version of Wallis' formula".............................................. 629

Takesi Isiwata, Correction: "Mappings and spaces" ................... 630

Henry B. Mann, Josephine Mitchell and Lowell Schoenfeld, Correction to:

"Properties of differential forms in $n$ real variables" .... . .

James Calvert, Correction to: "An integral inequality with applications to the

Dirichlet problem"............................. 